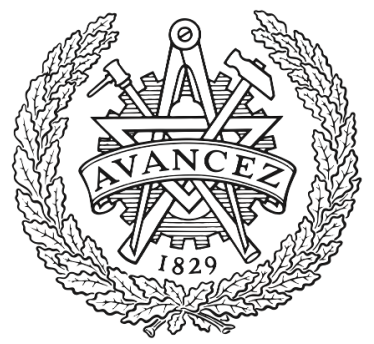

CHALMERS

UNIVERSITY OF TECHNOLOGY

\title{
Exploiting autonomous corner modules to resolve force constraints in the tyre contact patch
}

Downloaded from: https://research.chalmers.se, 2023-04-26 05:53 UTC

Citation for the original published paper (version of record):

Jonasson, M., Andreasson, J. (2008). Exploiting autonomous corner modules to resolve force constraints in the tyre contact patch. Vehicle System Dynamics, 46(7): 553-573. http://dx.doi.org/10.1080/00423110701504215

N.B. When citing this work, cite the original published paper. 


\title{
Exploiting autonomous corner modules to resolve force constraints in the tyre contact patch
}

\author{
MATS JONASSON*† and JOHAN ANDREASSON ${ }^{\ddagger}$ \\ ${ }^{\dagger}$ Chassis and Vehicle Dynamics, Volvo Car Corporation, SE-405 31 Göteborg, Sweden \\ ${ }^{\ddagger}$ KTH Vehicle Dynamics, SE-100 44 Stockholm, Sweden
}

\begin{abstract}
This paper presents a general force allocation strategy for over-actuated vehicles, utilising technologies where tyre forces can be more freely controlled than in conventional vehicles. For the purpose of illustration, this strategy has been applied and evaluated using a design proposal of an autonomous corner module (ACM) chassis during a transient open-loop response test. In this work, the vehicle has been forced to follow a trajectory, identical to the performance of a conventional front-steered vehicle during the manoeuvre studied. An optimisation process of tyre force allocation has been adopted along with tyre force constraints and cost functions to favour a desired solution. The vehicle response has been evaluated as open-loop, where tyre forces are shown to be allocated in a different manner than in conventional front-steered vehicles. A suggested approach for a control scheme of steering actuators is presented, where the actuator limitation is related to the lateral force possible. Finally, the force allocation strategy involves the ability to control vehicle slip independently from vehicle yaw rate. This opportunity has been adapted in the ACM vehicle in order to relax vehicle slip from the original trajectory description. In such circumstances, the ACMs demonstrate better utilisation of the adhesion potential.
\end{abstract}

Keywords: Autonomous corner module; Force allocation; Steering actuator; Tyre constraints; Vehicle control; Vehicle dynamics

\section{Introduction}

There is an increasing interest in vehicles where tyre forces can be individually controlled [1-5]. This class of technologies is able to perform new vehicle dynamics functions and better utilise the adhesion available in the tyre contact patch. Despite more than a century of development, motion control in conventional vehicles is restricted due to constraints in wheel kinematics. Given this situation, there is an interest to evaluate the performance of this new class of technology and relate it to the performance of a conventional vehicle.

In order to evaluate these two vehicle categories, the approach suggested is to subject these two vehicles to the transient open-loop response test with one period of sinusoidal input [6]. This test is commonly used by car manufacturers and a lot of studies have been undertaken using this specific test. The response from the transient open-loop response test indicates how well the vehicle is able to move in lateral direction, while maintaining vehicle stability. To fulfil demands and become even better, it is worth considering if chassis systems, where tyre forces can be individually controlled, could be an alternative solution. Furthermore, to avoid a specific strategy for handling different vehicle manoeuvres, it is attractive if the control strategy for normal driving conditions can be properly used even during extreme situations. One chassis solution, the autonomous corner module (ACM) [5,7], is considered in this paper, since the ACM is specifically designed to independently control longitudinal, lateral and vertical tyre forces.

This paper is organised as follows; section 2 presents the ACM design, section 3 describes motion control and the proposed approach of force allocation. Next, in section 4, the ACM vehicle is evaluated and compared with a conventional front-steered vehicle. Tyre and actuator modelling is formulated in section 5. In section 6 the trajectory is modified to independently control yaw rate and vehicle slip. Finally, this work is concluded in section 7 .

*Corresponding author. E-mail: mjonass2@volvocars.com; Tel: +46 31592918 


\section{The ACM chassis studied}

One chassis solution that allows tyre forces to be freely generated, and that is also designed to generate forces as closely as possible to the tyre contact patch, is the autonomous corner module (ACM) illustrated in figure 1.

This modularised concept involves electric linear steering actuators and an electric in-wheel motor to accomplish the generation of lateral and longitudinal tyre forces respectively. In order to control vertical forces, an upper control arm, regulated by an actuator in full active mode, is guided between wheel and car body. For more detailed descriptions, see $[5,7]$.
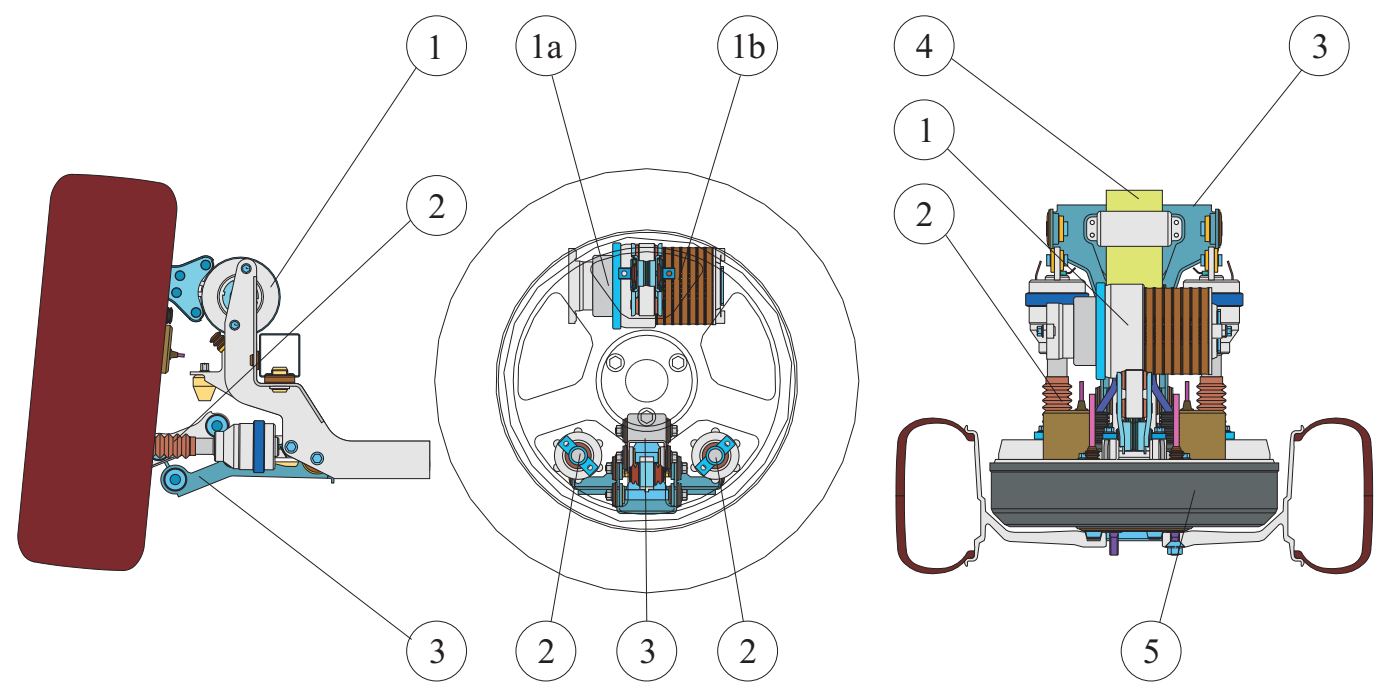

Figure 1. Design proposal for the ACM, showing front, side and top views. Vertical load actuator (1) with damper (1a) and adjustable spring (1b), steering actuators (2), lower arm (3), leaf spring (4) and in-wheel motor (5).

\section{Vehicle motion control}

This section formulates the proposed control principle as illustrated in figure 2. This is based on the assumption that a vehicle follows the state-space trajectory reference, which can be estimated from driver commands. Recent research findings suggest control methods where the vehicle controller has the trajectory errors as reference inputs [8]. Feedback control is of course needed to compensate for error and disturbance. However, the focus on this work is the open-loop behaviour, i.e. the immediate force allocation response for present vehicle states. The nomenclature used in this section is further described in appendix A.

\subsection{Vehicle motion}

If the actuators in the suggested concept are fully exploited, the number of vehicle force excitations exceeds the number of vehicle degrees of freedom and the vehicle becomes over-actuated [9].

Considering the vehicle as a rigid body with mass, $m$, the desired vehicle motion can be determined by the trajectory, $\boldsymbol{v}^{\mathrm{ref}}=\left[\begin{array}{llllll}v_{x} & v_{y} & v_{z} & \omega_{x} & \omega_{y} & \omega_{z}\end{array}\right]^{\mathrm{T}}$, containing the vehicle longitudinal speed, $v_{x}$, vehicle lateral velocity, $v_{y}$, vehicle vertical velocity, $v_{z}$, vehicle roll rate, $\omega_{x}$, vehicle pitch rate, $\omega_{y}$, and finally vehicle yaw rate, $\omega_{z}$, as illustrated in figure 3 .

\subsection{Global force generation}

The vehicle trajectory is generated by resulting forces acting on the centre of gravity, denoted as global

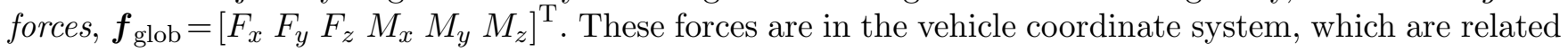




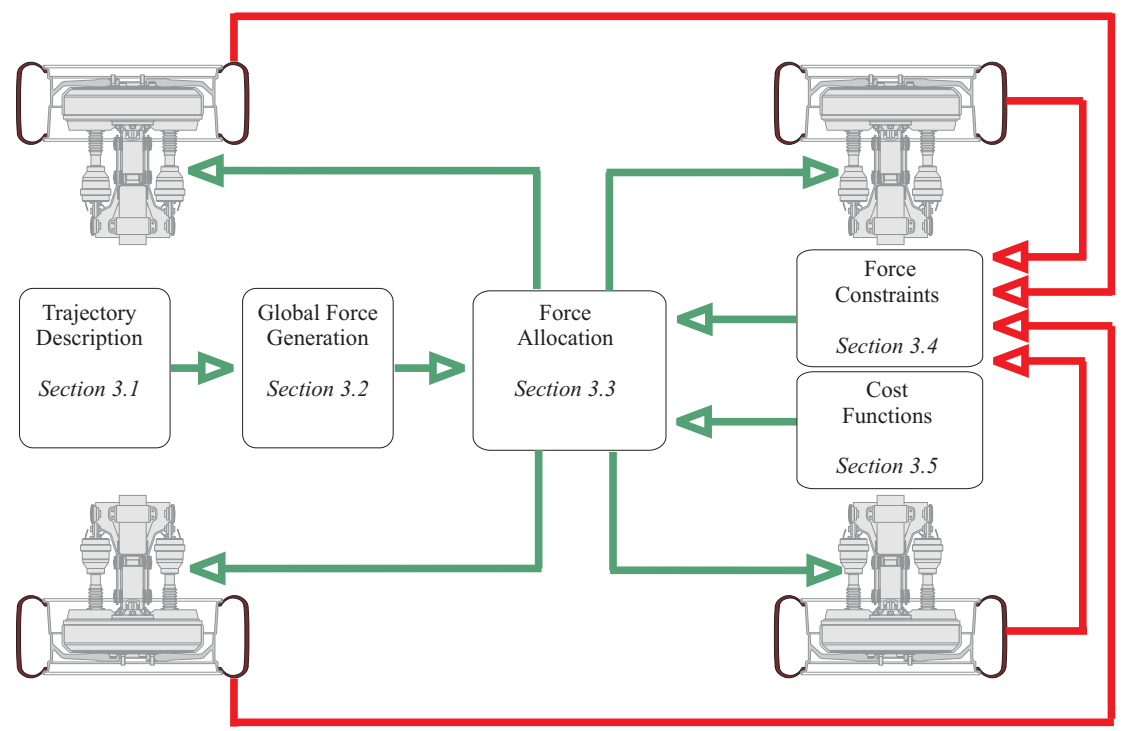

Figure 2. Procedure of force allocation and references to the subsections of section 3.

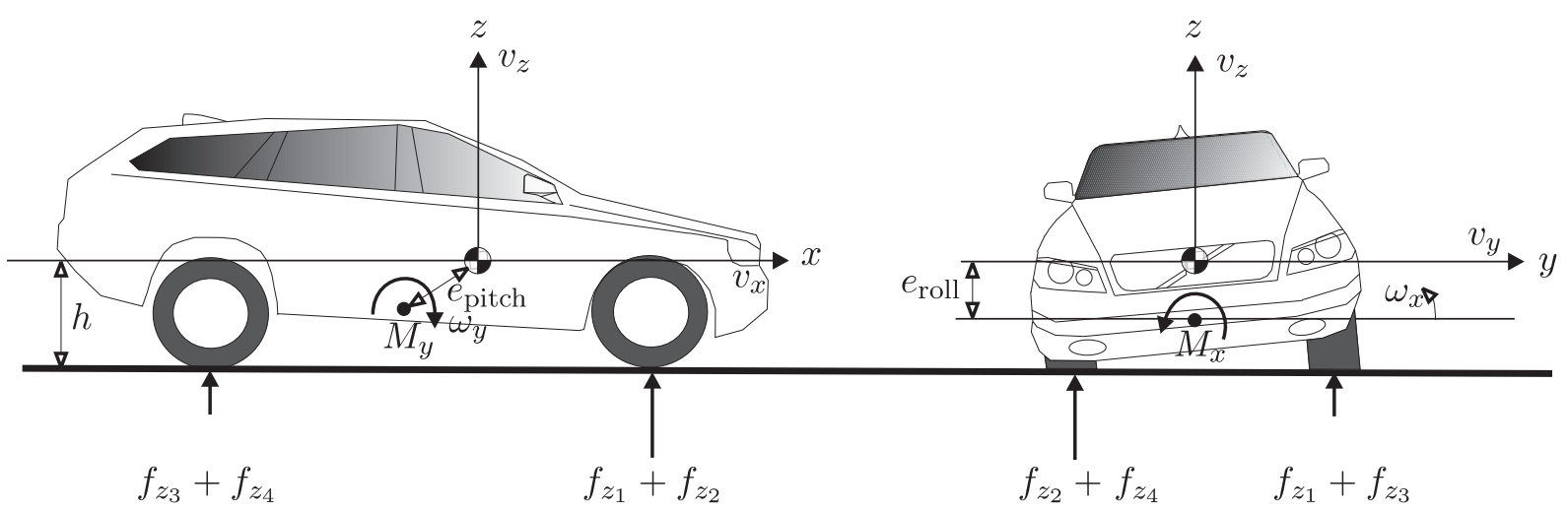

Figure 3. Side and front view of the vehicle studied, including parameters relevant for pitch and roll motions (the nomenclature list is shown in Appendix A).

to the trajectory as

$$
\boldsymbol{f}_{\text {glob }}=\left[\begin{array}{c}
F_{x} \\
F_{y} \\
F_{z} \\
M_{x} \\
M_{y} \\
M_{z}
\end{array}\right]=\left[\begin{array}{c}
m\left(\dot{v}_{x}-v_{y} \omega_{z}\right) \\
m\left(\dot{v}_{y}+v_{x} \omega_{z}\right) \\
m g \\
\left(I_{x x}-2 m_{w}\left(d_{1}^{2}+d_{2}^{2}\right)+e_{\mathrm{roll}}^{2} m\right) \dot{\omega}_{x} \\
\left(I_{y y}-2 m_{w}\left(d_{3}^{2}+d_{4}^{2}\right)+e_{\mathrm{pitch}}^{2} m\right) \dot{\omega}_{y} \\
I_{z z} \dot{\omega}_{z}
\end{array}\right]
$$

where the vertical excitation from road disturbance is intentionally ignored and the principal moments of inertia for the vehicle, $I_{x x}, I_{y y}, I_{z z}$, are given by the entries from the diagonal in the vehicle inertia tensor. The products of inertia, $I_{x y}, I_{y z}, I_{x z}$, are neglected. In addition, the wheels are assumed not to take part in the roll and pitch motions. Therefore, in equation (1), the vehicle inertias for roll and pitch motion are adjusted for the car body only, by removing the contributions from the unsprung masses, $m_{w}$.

The car body will roll and pitch around a roll and a pitch axis respectively. The inertias for roll and pitch motion, in equation (1), are hereby adjusted for rotations around axis displacements, $e_{\text {roll }}$ and $e_{\text {pitch }}$. It is also assumed that the height from the ground to the centre of gravity and to the roll and pitch axes are assumed to be constant, even during large executions of roll and pitch movements. This is a notable simplification for vehicles using conventional wheel suspensions, especially for the roll centre, which usually executes a significant nose-down movement (Front McPherson and rear multi-link). 


\subsection{Force allocation to the corners}

The global forces are related to the individual corner forces, $\boldsymbol{f}=\left[f_{x_{1}} f_{y_{1}} f_{z_{1}} \cdots f_{x_{4}} f_{y_{4}} f_{z_{4}}\right]^{\mathrm{T}}(i=1,2,3,4)$, given in the vehicle coordinate system as

$$
f_{\text {glob }}=\mathrm{D} f
$$

where

$$
\mathbf{D}=\left[\begin{array}{cccccccccccc}
1 & 0 & 0 & 1 & 0 & 0 & 1 & 0 & 0 & 1 & 0 & 0 \\
0 & 1 & 0 & 0 & 1 & 0 & 0 & 1 & 0 & 0 & 1 & 0 \\
0 & 0 & 1 & 0 & 0 & 1 & 0 & 0 & 1 & 0 & 0 & 1 \\
0 & h & d_{1} & 0 & h & -d_{2} & 0 & h & d_{1} & 0 & h & -d_{2} \\
-h & 0 & -d_{3} & -h & 0 & -d_{3} & -h & 0 & d_{4} & -h & 0 & d_{4} \\
-d_{1} & d_{3} & 0 & d_{2} & d_{3} & 0 & -d_{1} & -d_{4} & 0 & d_{2} & -d_{4} & 0
\end{array}\right]
$$

Here $d_{1}, d_{2}, d_{3}, d_{4}$ and $h$ represent vehicle dimensions as illustrated in figure 4 . Observe that equation (2) is under-determined and an infinite set of solutions of corner forces exist.

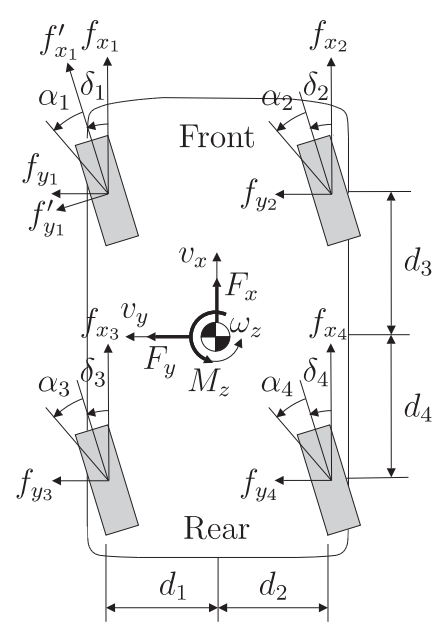

Figure 4. Vehicle forces in the horizontal plane.

\subsection{Force constraints in the tyre contact patch}

There are limitations to the magnitudes of tyre forces in the contact patch that can be generated. If tyre forces are not taken into consideration, the solution to equation 2 will be out of its expected range quite considerably. The maximum lateral tyre force possible is not only dependent on vertical load, $f_{z_{i}}$, and coefficient of friction, $\mu_{i}$, but also the longitudinal force limits the maximum lateral tyre force. Therefore, the tyre force constraint needs to be formulated in order to solve equation 13 further on in section 3.5. In this case, the tyre force constraint is modelled using an elliptic relation as

$$
\frac{\left(f_{x_{i}}^{\prime}\right)^{2}}{\left(f_{x_{i}}^{\prime \max }\right)^{2}}+\frac{\left(f_{y_{i}}^{\prime}\right)^{2}}{\left(f_{y_{i}}^{\prime \max }\right)^{2}} \leq 1
$$

where $f_{x_{i}}^{\prime}$ and $f_{y_{i}}^{\prime}$ denote tyre forces in the wheel coordinate system. The maximum tyre forces in longitudinal and lateral directions are denoted as $f_{x_{i}}^{\prime \text { max }}$ and $f_{y_{i}}^{\prime \text { max }}$ respectively. These are formulated as

$$
f_{x_{i}}^{\prime \max }\left(f_{z_{i}}, \mathrm{~d} f_{z_{i}}, \gamma_{i}\right)=f_{z_{i}} p_{x_{i}}, \quad f_{y_{i}}^{\prime \max }\left(f_{z_{i}}, \mathrm{~d} f_{z_{i}}, \gamma_{i}\right)=f_{z_{i}} p_{y_{i}},
$$




$$
\begin{gathered}
p_{x_{i}}=\mu_{i}\left(k_{x 1}-k_{x 2} \mathrm{~d} f_{z_{i}}\right)\left(1+k_{x 3} \gamma_{i}+k_{x 4} \gamma_{i}^{2}\right), \quad p_{y_{i}}=\mu_{i}\left(k_{y 1}-k_{y 2} \mathrm{~d} f_{z_{i}}\right)\left(1+k_{y 3} \gamma_{i}+k_{y 4} \gamma_{i}^{2}\right) \quad \text { and } \\
\mathrm{d} f_{z_{i}}=\frac{f_{z_{i}}-f_{z, \text { nom }}}{f_{z, \text { nom }}} .
\end{gathered}
$$

Here, the wheel camber is represented by $\gamma_{i}$. In addition, $k_{x 1}, \ldots, k_{x 4}, k_{y 1}, \ldots, k_{y 4}$ and $f_{z \text {,nom }}$ are tyre parameters for the $i$ th tyre. Vertical tyre forces are restricted to be within magnitudes that the wheel suspension can withstand.

When equation (2) is to be solved, the constraint formulated in equation (4) must be taken into account to secure all tyre forces, $\boldsymbol{f}^{\prime}$, to be within these limits. However, optimisation with non-linear limitations are time-consuming, which can make it difficult to allocate forces on-board. Thus, to make the optimisation calculation more rapid and to facilitate the use of a standard solver, the non-linear tyre force limitations are here approximated by using linearisation. Consider the tyre force limitation for each tyre as a surface in $\mathbb{R}^{3}$ :

$$
a\left(f_{x_{i}}^{\prime}, f_{y_{i}}^{\prime}, f_{z_{i}}\right)=\frac{\left(f_{x_{i}}^{\prime}\right)^{2}}{p_{x_{i}}^{2}}+\frac{\left(f_{y_{i}}^{\prime}\right)^{2}}{p_{y_{i}}^{2}}-f_{z_{i}}^{2}=0 .
$$

As seen, this surface resembles an elliptical cone (if the dependencies of $\mathrm{d} f_{z_{i}}$ in $p_{x_{i}}$ and $p_{y_{i}}$ are disregarded). An approximation of this surface can be described by a number, $n$, on the tangent plane, where each plane, $k$, has a normal vector, $\nabla \boldsymbol{a}_{k}=\left[\frac{\partial a_{k}}{\partial f_{x_{i}}^{\prime}} \frac{\partial a_{k}}{\partial f_{y_{i}}^{\prime}} \frac{\partial a_{k}}{\partial f_{z_{i}}}\right]$, and a scalar, $b_{k}$. The elements in $\nabla \boldsymbol{a}_{k}$ regard partial derivatives calculated for points that satisfy equation (8). The graphical representation of the tyre constraints is shown in figure 5 . By dividing the surface piecewise into a set, $n$, of linear planes, the tyre constraints can finally be formulated as

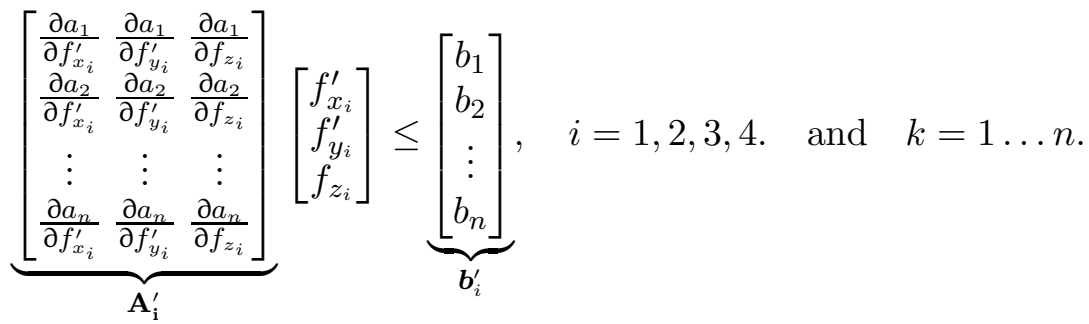

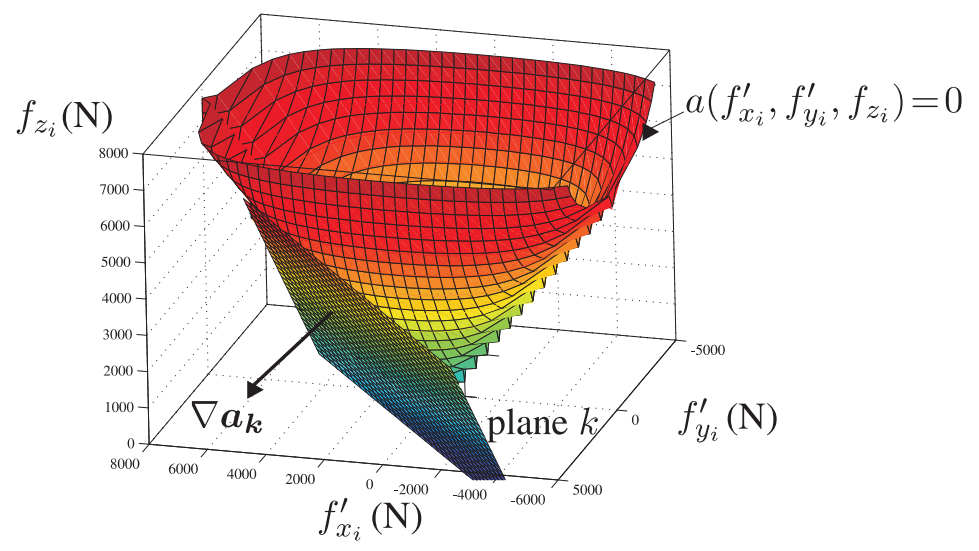

Figure 5. Tyre force constraints approximated by $n$ planes.

However, the linearised tyre force constraint, $\mathbf{A}_{\mathbf{i}}^{\prime}$, is valid in the tyre force coordinate system (which is indicated by the ' sign). By rotating it using the wheel steering angle, $\delta_{i}$, the constraint can be re-expressed 
in the vehicle coordinate system instead by using the relation, $\left[f_{x_{i}}^{\prime} f_{y_{i}}^{\prime} f_{z_{i}}\right]^{\mathrm{T}}=\mathbf{T}_{i}\left[f_{x_{i}} f_{y_{i}} f_{z_{i}}\right]^{\mathrm{T}}$, where

$$
\mathbf{T}_{\mathbf{i}}=\left[\begin{array}{ccc}
\cos \delta_{i} & \sin \delta_{i} & 0 \\
-\sin \delta_{i} & \cos \delta_{i} & 0 \\
0 & 0 & 1
\end{array}\right], \quad i=1,2,3,4
$$

Consequently, the constraints can further be derived for all corners as

$$
\operatorname{AT} f \leq b,
$$

where $\mathbf{A}=\operatorname{diag}\left[\mathbf{A}_{\mathbf{1}}, \mathbf{A}_{\mathbf{2}}, \mathbf{A}_{\mathbf{3}}, \mathbf{A}_{\mathbf{4}}\right], \mathbf{T}=\operatorname{diag}\left[\mathbf{T}_{\mathbf{1}}, \mathbf{T}_{\mathbf{2}}, \mathbf{T}_{\mathbf{3}}, \mathbf{T}_{\mathbf{4}}\right]$ and $\boldsymbol{b}=\left[\boldsymbol{b}_{1}, \boldsymbol{b}_{2}, \boldsymbol{b}_{3}, \boldsymbol{b}_{4}\right]^{\mathrm{T}}$.

\subsection{Solution of force allocation formulation involving cost functions}

High magnitude corner force generation is related to high energy consumption, over-dimensioned actuators and significant tyre wear. Given this background, it is attractive to keep corner forces as low as possible. Therefore, cost functions are introduced to avoid deviations of forces from nominal levels. One way to accomplish this, is to assume vertical tyre forces to be close to static load and longitudinal and lateral tyre forces to be kept close to a quarter of the requested global forces in question. The latter are also weighted with the friction coefficient and the vertical load, to ensure the adhesion potential to be better assigned at each tyre, i.e. higher vertical load along with large friction coefficient enables the generation of high longitudinal and lateral forces respectively. The proposed cost functions are expressed as

$$
\boldsymbol{f}_{\text {cost }}=\left[\frac{\mu_{1} f_{z_{1}} F_{x}}{m g \mu_{\Sigma}} \frac{\mu_{1} f_{z_{1}} F_{y}}{m g \mu_{\Sigma}} \frac{m g d_{2}}{2\left(d_{1}+d_{2}\right)} \cdots \frac{\mu_{4} f_{z_{4}} F_{x}}{m g \mu_{\Sigma}} \frac{\mu_{4} f_{z_{4}} F_{y}}{m g \mu_{\Sigma}} \frac{m g d_{1}}{2\left(d_{1}+d_{2}\right)}\right]^{\mathrm{T}}, \mu_{\Sigma}=\sum_{i=1}^{4} \mu_{i} \text {. }
$$

By incorporating these cost functions into equation (2), the rank is increased and the equations are turned into an over-determined equation system, which can now be computed by solving the following constrained, linear, least-square optimisation problem:

$$
\min _{\boldsymbol{f}} \frac{1}{2}\left\|\mathbf{W}\left[\begin{array}{c}
\mathbf{D} \\
\mathbf{I}
\end{array}\right] \boldsymbol{f}-\mathbf{W}\left[\begin{array}{c}
\boldsymbol{f}_{\text {glob }} \\
\boldsymbol{f}_{\text {cost }}
\end{array}\right]\right\|_{2}^{2}, \quad \mathbf{A T} \boldsymbol{f} \leq \boldsymbol{b}
$$

In equation (13), $\mathbf{W}=\operatorname{diag}\left(w_{1}, w_{2}, \ldots\right)$ is added in order to prioritise each row in the optimisation formulation.

\section{Evaluation of two vehicle configurations}

In order to evaluate the proposed force allocation method, the ACM vehicle will be subjected to a standardised test procedure; ISO/TR 8725, transient open-loop response test method with one sinusoidal input [6]. The parameters for the ACM vehicle are shown in table 1.

The aim of this test is to determine the transient response of a vehicle subjected to one period of sinusoidal steering input of $1 / 2 \mathrm{~Hz}$ to the steering wheel. However, the ACM vehicle uses steer-by-wire, which differs from a conventional front-steered vehicle with mechanical rack steering. In the absence of a mechanical connection between the steering wheel and the resulting trajectory in the ACM vehicle, transfer function characteristics will be dependent on actual controller design, which makes it hard to measure stability. To overcome this dilemma, the approach suggested is to force the ACM vehicle to follow the trajectory, which in turn, can be derived from a conventional front-steered vehicle. This has been carried out in two ways; firstly, measurements on a conventional front-steered vehicle on a test track using a steering machine and sensors for vehicle states and secondly, by performing computer simulations for the same vehicle using 
ADAMS $/$ car $^{1}$ with a very high resolution of sub-assemblies (model involving approximately 700 degrees of freedom).

The parameters for the conventional front-steered vehicle are found in table 1 (including those for the ACM vehicle). In this research, both vehicles have been given the same set of parameters in order to justify the examination of force allocation characteristics for the two vehicles under consideration. The ACM vehicle model is implemented using MATLAB/Simulink ${ }^{1}$. The road friction coefficients during measurements are estimated to be approximately $\mu_{i}=1.0$, which also have been used for the computer simulations. The wheel camber angles, $\gamma_{i}$, used for both vehicles are $-0.4^{\circ}$.

\begin{tabular}{lccc} 
Table 1. Vehicle model parameters. & & & \\
\hline Parameter & Symbol & Value & Unit \\
\hline Vehicle mass & $\mathrm{m}$ & 2200 & $\mathrm{~kg}$ \\
Vehicle dimensions & $d_{1}, d_{2}, d_{3}, d_{4}$ & $1.3,1.5,0.8,0.8$ & $\mathrm{~m}$ \\
Height of centre of gravity & $h$ & 0.66 & $\mathrm{~m}$ \\
Principal moments of inertia & $I_{x x}, I_{y y}, I_{z z}$ & $850,4200,4561$ & $\mathrm{kgm}^{2}$ \\
Distance between roll-axis and centre of gravity & $e_{\text {roll }}$ & 0.51 & $\mathrm{~m}$ \\
Distance between pitch-axis and centre of gravity & $e_{\text {pitch }}$ & 0.35 & $\mathrm{~m}$ \\
Unsprung mass & $m_{w}$ & 55 & $\mathrm{~kg}$ \\
Tyre radius (235/65 R17) & $r$ & 0.37 & $\mathrm{~m}$ \\
Tyre parameters & $c_{1}, c_{2}, f_{z, \mathrm{nom}}$ & 19.301 .705355 & \\
Tyre parameters & $k_{x 1}, k_{x 2}$ & 1.120 .130 & 1.000 .155 \\
Tyre parameters & $k_{y 1}, k_{y 2}$ & 1.000 .155
\end{tabular}

a The moment of inertias originate in the vehicle centre of gravity and are parallel with the ground coordinate system.

\subsection{Test response from the conventional front-steered vehicle}

The manoeuvre studied involves a lane change at a vehicle velocity of $80 \mathrm{~km} / \mathrm{h}$. Figure $6 \mathrm{a}$ shows the measured and simulated steering wheel angle of the conventional front-steered vehicle, where the magnitude of the steering wheel angle has been adjusted to the maximum lateral acceleration achieved. The response of the vehicle, i.e. the resulting trace for the centre of gravity is shown in figure $6 \mathrm{~b}$. As seen, the roll angle and the lateral acceleration are clearly related to each other, which is shown in figure 7a. It is interesting to note the wheel entrance into the wheel suspension bump stop (after $2.2 \mathrm{~s}$ ), where the gradient of the roll angle rapidly changes and the lateral force is maximised. Figure $7 \mathrm{~b}$ indicates what is typical for frontsteered vehicles; the time lag between front and rear axle slip. As previously discussed, the response of

(a) Steering wheel angle

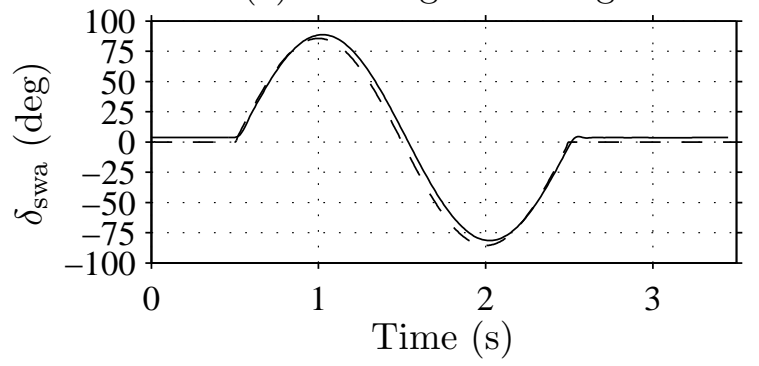

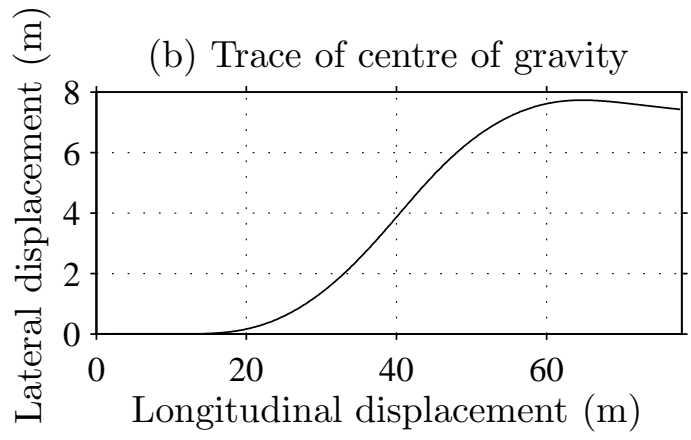

Figure 6. a) Measured (solid line) and simulated (dashed line) steering wheel angle as a vehicle input. (b) Vehicle trace (from ADAMS/car simulation).

the conventional front-steered vehicle during this test is used to define vehicle trajectory. As indicated in figure 8, results from measurements and ADAMS/car computer simulations are close to each other, with only minor differences in the states presented. Since additional information about actual tyre forces is

\footnotetext{
${ }^{1}$ ADAMS is a registered trademark of the MSC Software Corporation or its subsidiaries.

${ }^{1}$ MATLAB and Simulink are registered trademarks of The Mathworks, Inc., Natick, Massachusetts, U.S.A.
} 

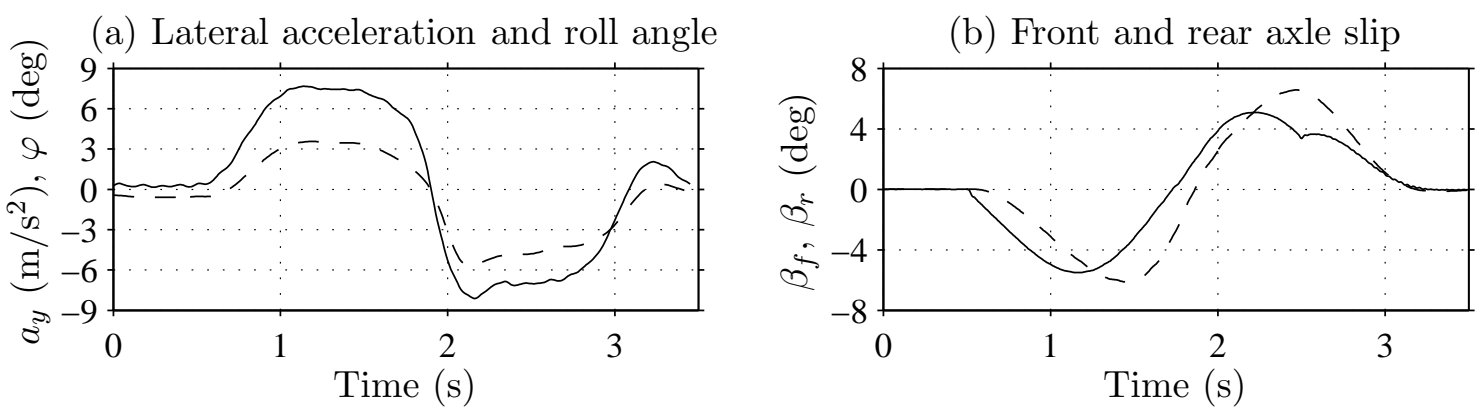

Figure 7. a) Lateral acceleration (solid line) and roll angle (dashed line) measured on the conventional front-steered vehicle studied. b) Front (solid line) and rear axle (dashed line) slip from ADAMS/car simulation (no measurements available).

given from the ADAMS/car computer simulation, in conjunction with the measurement similarities, the trajectory from the ADAMS/car computer simulation is selected as the reference.

(a) Vehicle velocity

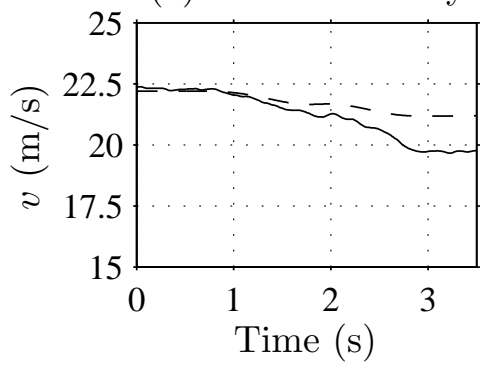

(b) Vehicle lateral acceleration

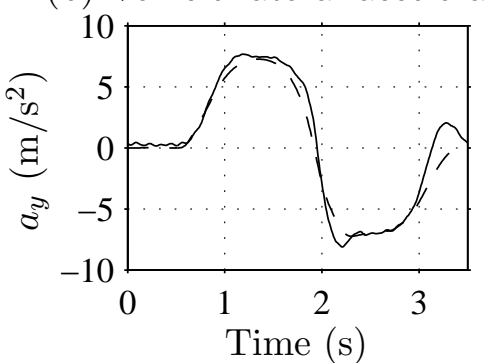

(c) Vehicle yaw rate

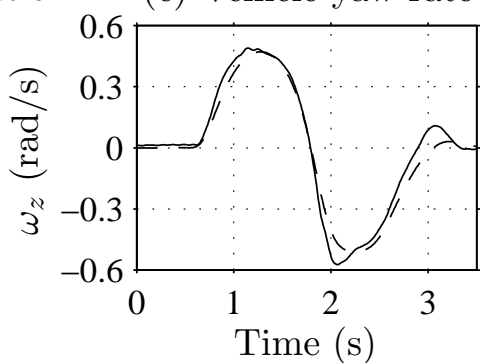

Figure 8. Part of trajectory from the conventional front-steered vehicle. Solid lines: Measurements by an automatic steering machine. Dashed lines: Results from ADAMS/car computer simulation.

\subsection{Tyre forces}

Figure 9 shows the vertical forces and load transfer present. The load transfer between the tyres in the conventional front-steered vehicle is in essence determined by the anti-roll bars (front-end distribution) in conjunction with differences in spring and damper stiffness. As previously discussed, the ACM vehicle is also able to allocate the vertical forces freely, which may result in another load transfer pattern. In this research, the vertical corner forces in the ACM vehicle are assumed to be equal to the vertical tyre forces, i.e. the vertical dynamics of the unsprung mass is intentionally omitted. Naturally, this is a simplification especially for vehicles with notable in-wheel motor weights, which contribute to the unsprung mass. Even if the unsprung mass can be controlled, there will be a compromise between tyre grip and body control.

At a first glance, figures 9 and 10 indicate similar tyre forces for both vehicle configurations. Since the ACM vehicle is forced to follow the trajectory, which in turn, has been determined from a front-steered vehicle, the force allocation is similar. However, there are minor differences in the tyre forces for the vehicle configurations in question, partly arising from inaccuracy and assumptions in vehicle modelling, but also from the utilisation of the resolved tyre force constraints combined with the cost functions adopted as previously described in sections 3.4 and 3.5 respectively. From figure 11, it is clear that longitudinal tyre forces are used to generate an additional yaw moment. The in-wheel motors contribute to a third of the total desired yaw moment. For this specific trajectory, there is a great deal of global lateral force, which in turn, gives small longitudinal tyre forces compared with the lateral ones.

The use of additional longitudinal tyre forces combined with lateral ones, implies another utilisation of the tyre adhesion potential than would be obtained for lateral tyre forces only $[4,10]$. Here, this adhesion 

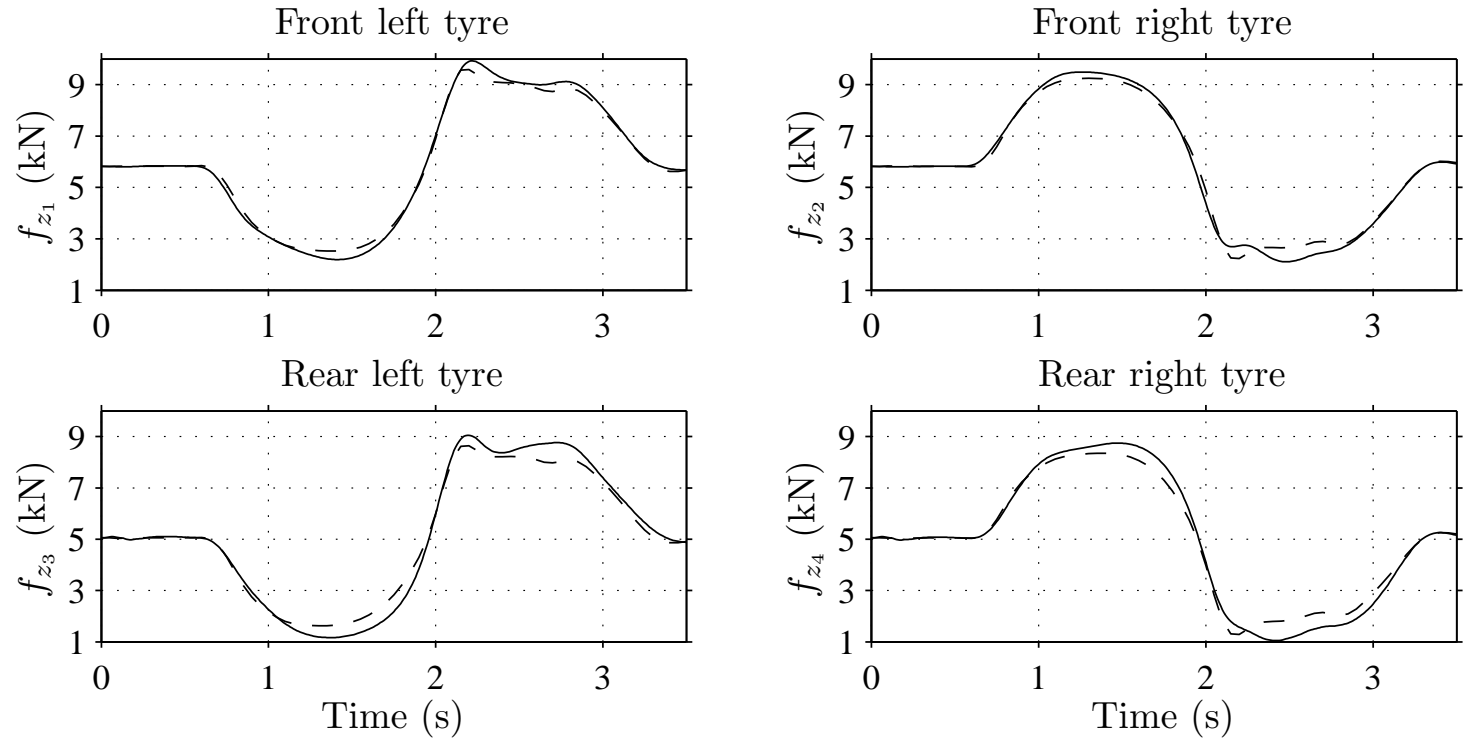

Figure 9. Vertical forces arising from load transfer in the conventional front-steered vehicle (solid lines) and vertical forces from force allocation in the ACM vehicle (dashed lines).
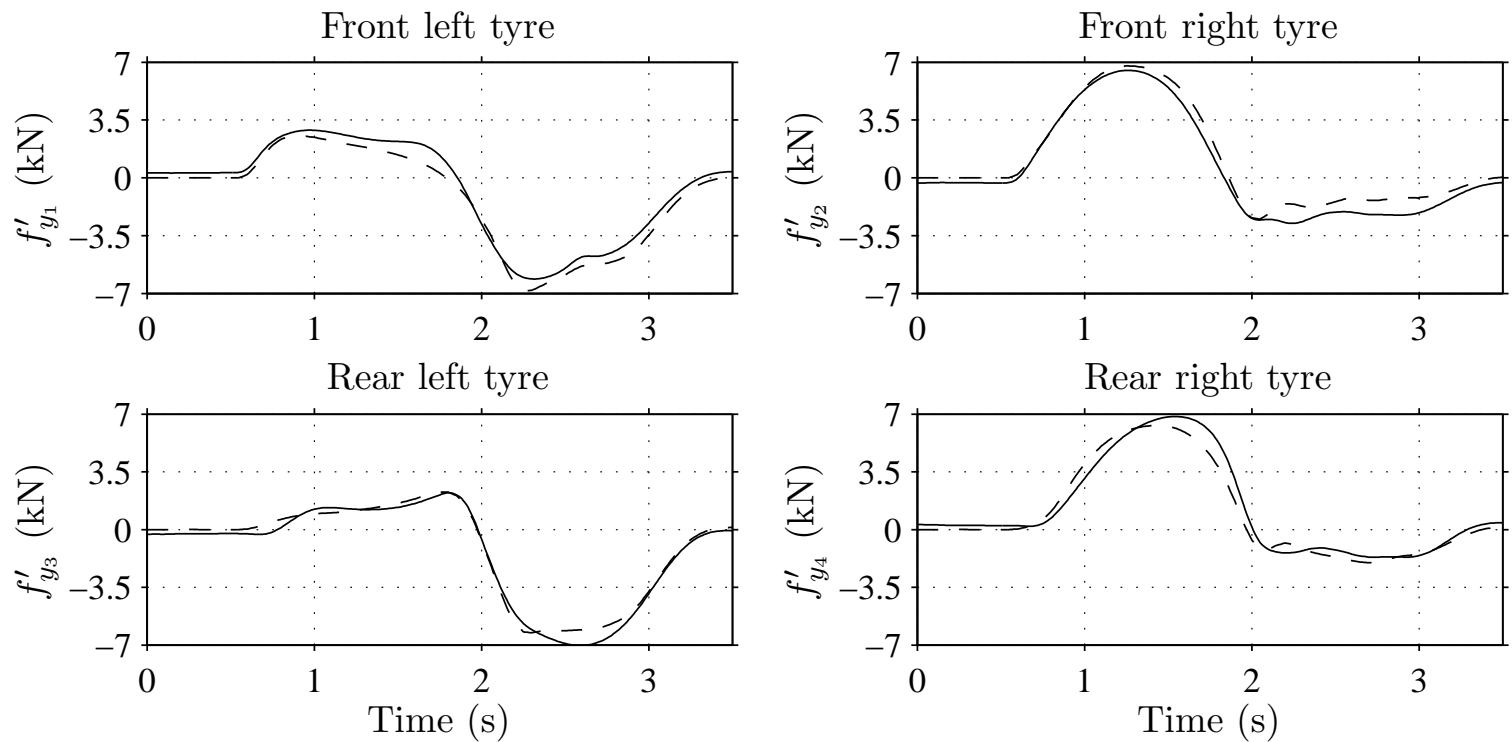

Figure 10. Lateral tyre forces arising from load transfer in the conventional front-steered vehicle (solid lines) and lateral forces from force allocation in the ACM vehicle (dashed lines).

potential is formulated as

$$
\eta_{i}=\sqrt{\frac{\left(f_{x_{i}}^{\prime}\right)^{2}}{\left(f_{x_{i}}^{\prime \max }\right)^{2}}+\frac{\left(f_{y_{i}}^{\prime}\right)^{2}}{\left(f_{y_{i}}^{\prime \max }\right)^{2}}} .
$$

From figure 12 it is evident that the adhesion potential, as formulated in equation 14, is better used. As seen, the conventional front-steered vehicle partly reaches high levels, while the ACM vehicle tends to spread adhesion potential more evenly. This effect partly comes from a low generation of lateral forces when the magnitudes of vertical tyre forces are small. In addition, the generation of small longitudinal forces (compared with lateral tyre forces), contributes positively to the yaw moment, without loosing too much adhesion potential. However, when the vertical tyre forces are low, the adhesion potentials for both 


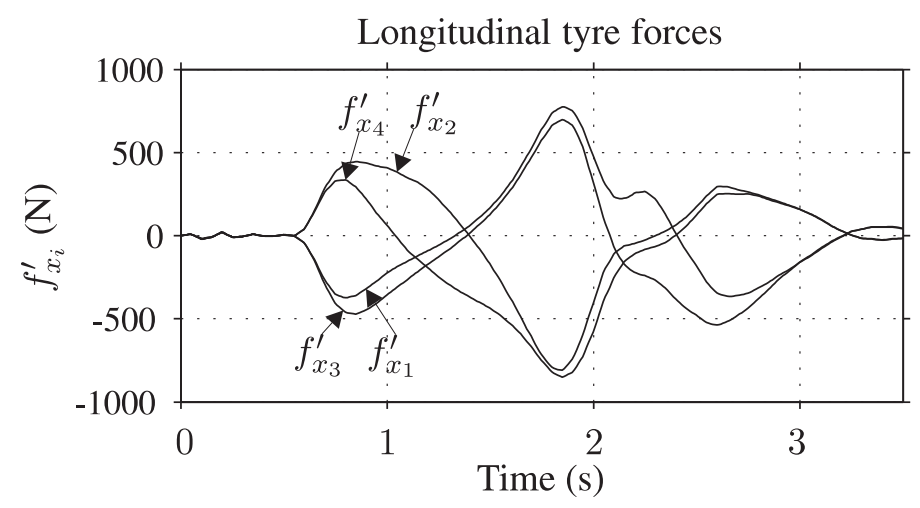

Figure 11. Longitudinal tyre forces from the applied force allocation in the ACM vehicle.

vehicle configurations are essentially equal. To evaluate the utilisation of all tyres' adhesion potentials, the vehicle adhesion potential, here defined as the mean value $\eta_{\text {mean }}=\frac{1}{4} \sum_{i=1}^{4} \eta_{i}$ of the tyre potentials, is introduced. From figure 13 it is evident that the vehicle adhesion potential is significantly lower for the ACM vehicle.
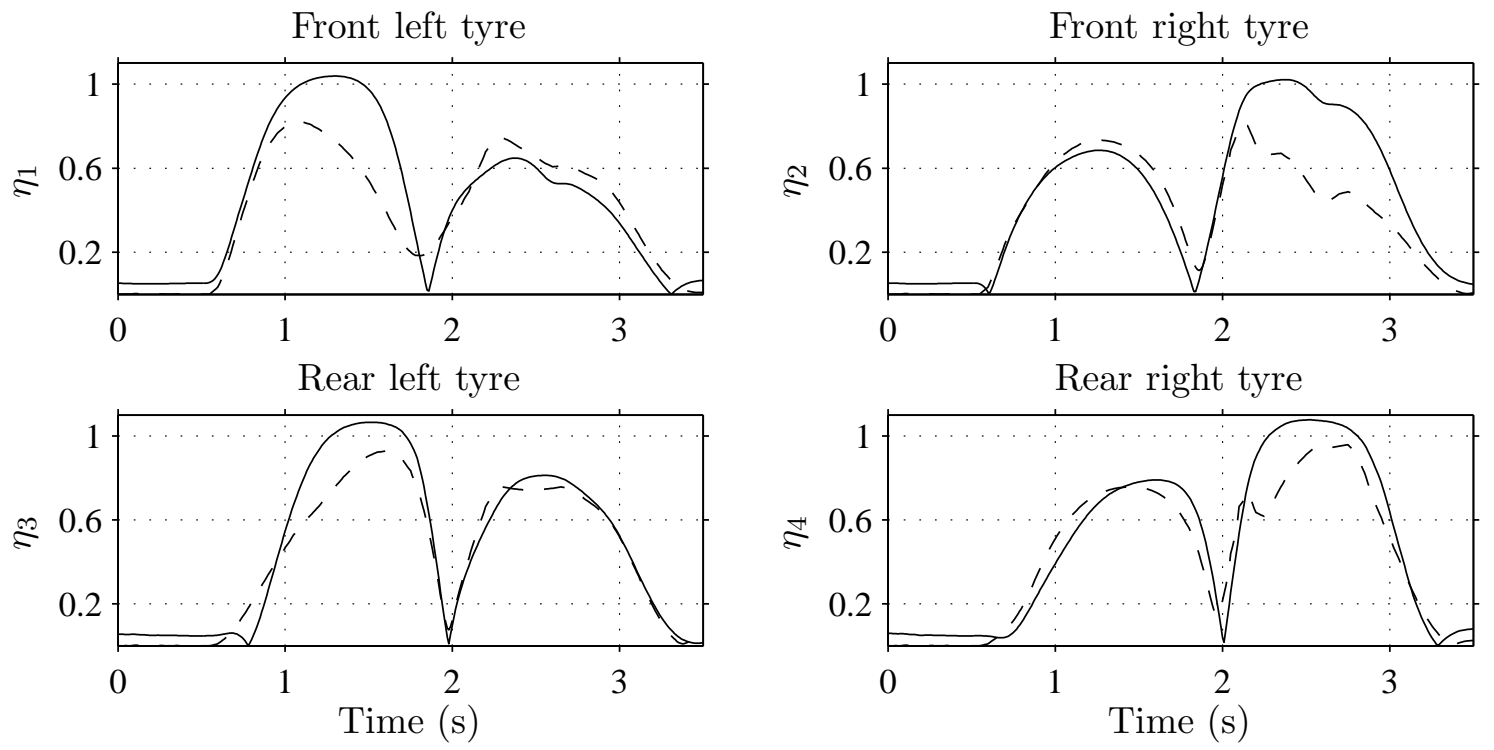

Figure 12. Adhesion potential for the conventional front-steered vehicle (solid line) and the ACM vehicle (dashed line).

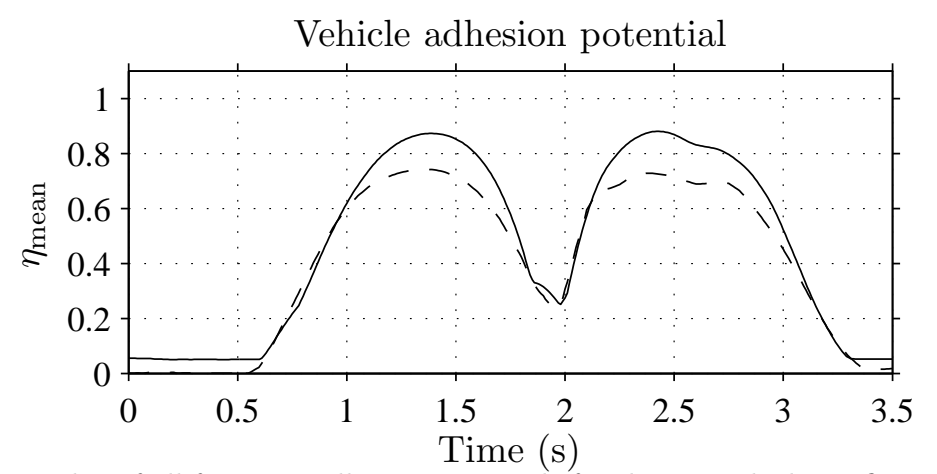

Figure 13. Mean value of all four tyre adhesion potentials for the two vehicle configurations in figure 12. 


\section{Tyres and actuators in the ACM vehicle}

This section formulates tyre and steering actuator models. These models are used to compile the steering effort needed and implications of the actuator limitation, and in section 6 , to re-express lateral forces into steering angles.

The procedure of force allocation, as described in section 3.5, distributes forces to each corner of the vehicle. This is followed by corner forces being transformed to tyre forces in the wheel coordination system. To be able to assign forces to the actuators, the relation between tyre slip and tyre forces must be known; this relation is known as the inverse tyre model. The longitudinal tyre force and the lateral side slip, can, in turn, be used to control the in-wheel motor torque and the steering-actuator positions respectively. The assignment of actuator forces for each ACM is illustrated in figure 14.

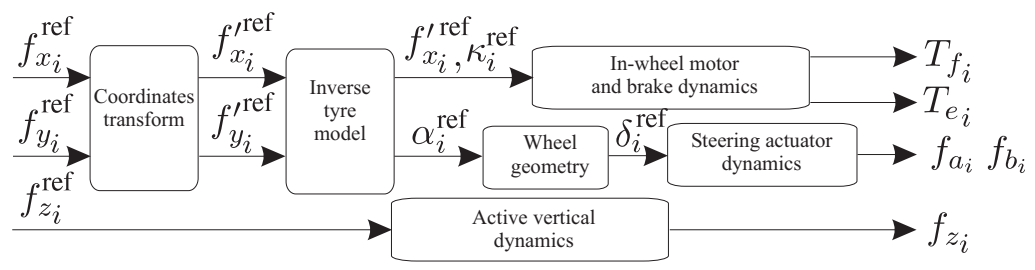

Figure 14. Force assignment to the ACM actuators (see nomenclature used in appendix A).

\section{$5.1 \quad$ Tyre modelling}

In this case, the use of an inverse tyre model is derived from a shortened variant of the well-known magic formula [11] as

$$
\begin{gathered}
\alpha_{i}=-\frac{1}{B_{y_{i}}} \tan \left[\frac{\arcsin \left(f_{y_{i}}^{\prime} / D_{y_{i}}\right)}{C_{y}}\right], \quad 0 \leq\left|f_{y_{i}}^{\prime}\right|<D_{y_{i}} \quad \text { and } \\
D_{y_{i}}=f_{y_{i}}^{\prime \max } \sqrt{1-\left(\frac{f_{x_{i}}^{\prime}}{f_{x_{i}}^{\prime \max }}\right)^{2}},
\end{gathered}
$$

where the maximum side slip allowed for equation $(15), \alpha_{i, \max }$, is

$$
\alpha_{i, \max }=-\frac{\operatorname{sign}\left(f_{y_{i}}^{\prime}\right)}{B_{y i}} \tan \left(\frac{\pi}{2 C_{y}}\right)
$$

The inverse given by equation (17) limits the side slip angle to $\alpha_{i, \max }$. Thus, the tyre forces are restricted to be within the positive gradient of the slip-force graphs [11]. The shape factor, $C_{y}$, and the stiffness factor, $B_{y_{i}}$, are magic formula variables, and $\alpha_{i}$ is the tyre side slip angle for the $i$ th tire. The peak value and stiffness factor are found as

$$
B_{y_{i}}=C_{F \alpha_{i}} /\left(C_{y} D_{y_{i}}\right), \quad C_{F \alpha_{i}}=c_{1} f_{z, \text { nom }} \sin \left[2 \arctan \left(\frac{f_{z_{i}}}{c_{2} f_{z, \text { nom }}}\right)\right]
$$

where $k_{z_{1}}, k_{z_{2}}, f_{z, \text { nom }}, c_{1}$ and $c_{2}$ are tyre parameters (see table 1 ). The side slip angle is compiled from the steering angle, $\delta_{i}$, as

$$
\alpha_{i}=\arctan \left(\frac{v_{y}+g_{i} \omega_{z}}{v_{x}}\right)-\delta_{i}, \quad i=1,2,3,4
$$


where $g_{1}=g_{2}=d_{3}$ and $g_{3}=g_{4}=-d_{4}$.

\section{$5.2 \quad$ Steering actuators}

In this case, there are two steering actuators present, according to figure 1, assumed to work symmetrically (i.e actuator transversal displacements are equal).

5.2.1 Steering actuator modelling and limitations. The steering actuators are screw types and are electrically driven, where the steady-state velocity of the linear piston is strongly dependent on the load applied to it as shown in figure 15. The measurements according to this figure demonstrate that the maximum steady-state speed of the piston decreases when the load increases. This response justifies adding a damping coefficient to the actuator model and, in turn, limit the steady-state velocity during force generation. This model is commonly used for modelling electrical machines.

$$
f_{\text {act }}-f_{\text {load }}=m_{\text {act }} \frac{\mathrm{d} v_{\text {act }}}{\mathrm{d} t}+b_{1} v_{\text {act }}
$$

where $f_{\text {act }}$ and $f_{\text {load }}$ represent the actuator force and load on it. The equivalent mass, $m_{\text {act }}$, of the piston moves with a velocity, $v_{\text {act }}$, and $b_{1}$ is the damping coefficient. According to figure 15 , this model forces

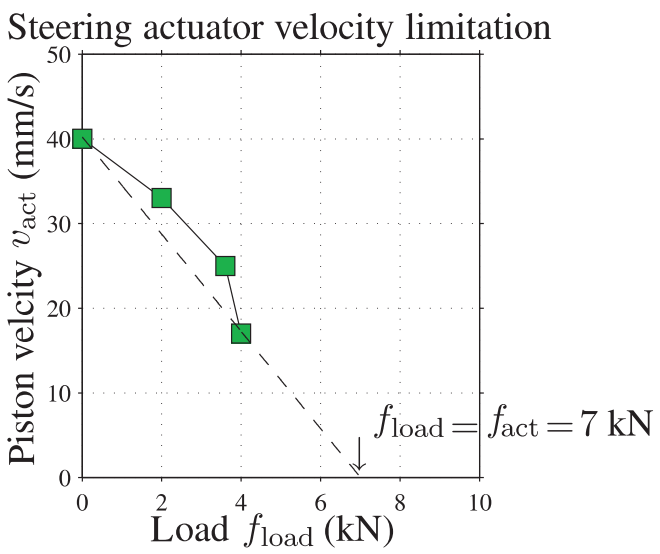

Figure 15. Speed limitation of the steering actuator in steady-state. Markers show measurements and the dashed line shows the limit according to the model.

the steering actuator to operate below the dashed line in steady-state conditions. However, the measured data in figure 15 indicates piston velocity to be exponentially degraded for high loads, possibly because of impact from friction, which, in turn, justifies a better model.

5.2.2 Steering actuator control. To improve robustness against disturbances arising from external wheel forces to the steering actuator, the design of the controller is chosen to be of type internal mode control $[12,13]$, which provides the opportunity to set a first-order closed-loop response and avoid undesired overshoots. In addition, the bandwidth can be determined and power consumption can be kept low. However, this type of control relies on an acceptable estimate of $b_{1}$ and deviations of it is critical if proper control is required.

The process of transporting the piston to a reference position is very slow compared to the speed process. The use of cascade control [14], where one outer loop controls the piston position and one inner loop controls the speed, is justified as it further improve robustness from disturbances from actuator loads. This is illustrated in figure 16. Furthermore, the maximum actuator force, $f_{\text {act }}$, implies the need for windup strategy in the speed regulator. 


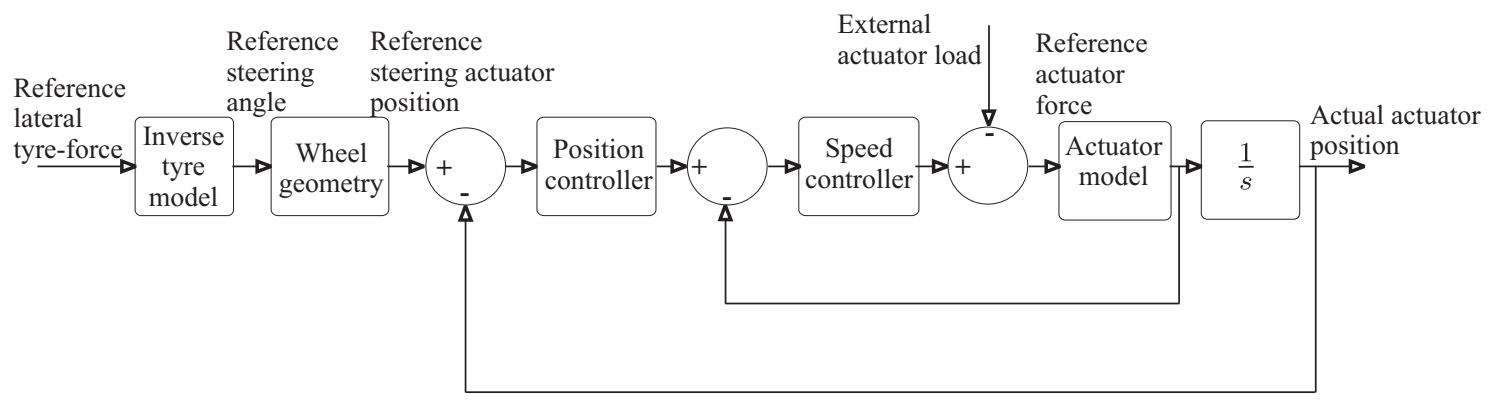

Figure 16. Control flowchart of the steering actuator.

5.2.3 Steering actuator force needed. As previously discussed, the loads on the steering actuators influence the steady-state speed possible, and in turn limit the lateral tyre force needed. For a better understanding of this influence, it could be of interest to estimate these loads and the impact of the steering actuator dynamics. From figure 17, the definition of wheel suspension forces can be identified. The lower control arm relieves the longitudinal pressure arising from longitudinal tyre loads and in turn, the steering actuator load acts along the linear movement of them.

The vertical tyre force causes an overturning moment, rotating the wheel top inwards. However, the spring and the lower control arm pull the wheel back until a moment of equilibrium is established. Hence, the impact of steering actuator loads arising from the vertical tyre force can be ignored. Given this situation,

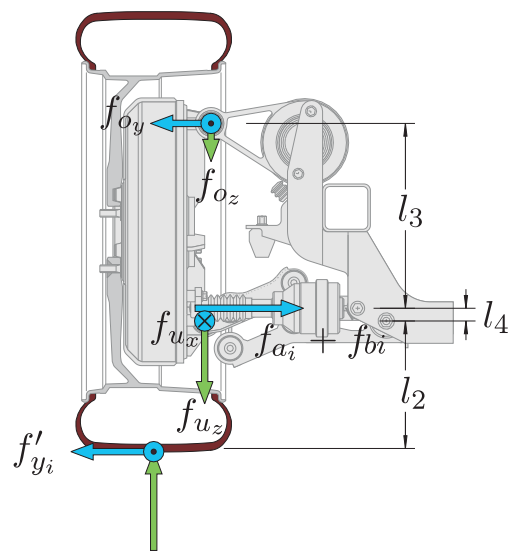

$f_{z_{i}}$

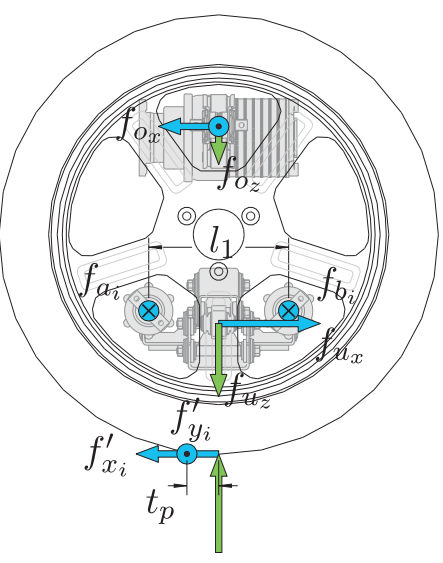

$f_{z_{i}}$

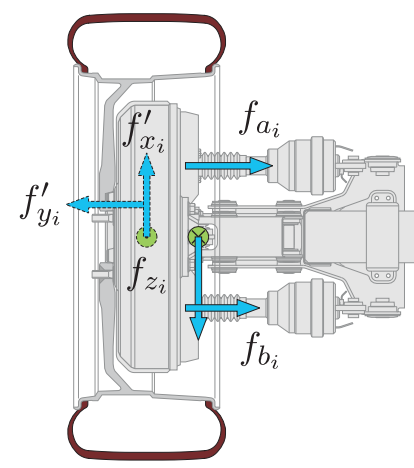

Figure 17. Wheel geometry and forces.

the loads on the two steering actuators, $f_{a_{i}}$ and $f_{b_{i}}$, are only dependent on lateral tyre force and pneumatic trail, $t_{p}$, as

$$
f_{a_{i}}=f_{y_{i}}^{\prime}\left(\frac{t_{p}}{l_{1}}+\frac{1}{2}+\frac{l_{2}}{2 l_{3}}\right), \quad f_{b_{i}}=f_{y_{i}}^{\prime}\left(-\frac{t_{p}}{l_{1}}+\frac{1}{2}+\frac{l_{2}}{2 l_{3}}\right) .
$$

Figure 18 shows the forces required for one of the steering actuators (see table 2 for the parameters used) in the front left wheel (worst case in terms of high force needed). Since the acceleration of the piston can be very high $\left(\frac{m_{\text {act }}}{b_{1}} \ll 1\right.$ and in case of $\left.f_{\text {act }}>f_{\text {load }}\right)$, the force gradient can be of a high magnitude. As seen, there is a positioning error present, which, in turn, gives deviation of lateral forces, and consequently, deviation of the trajectory. Clearly, the steering actuator is undersized, which motivates upgrading it in order to fulfil the specification needed. 

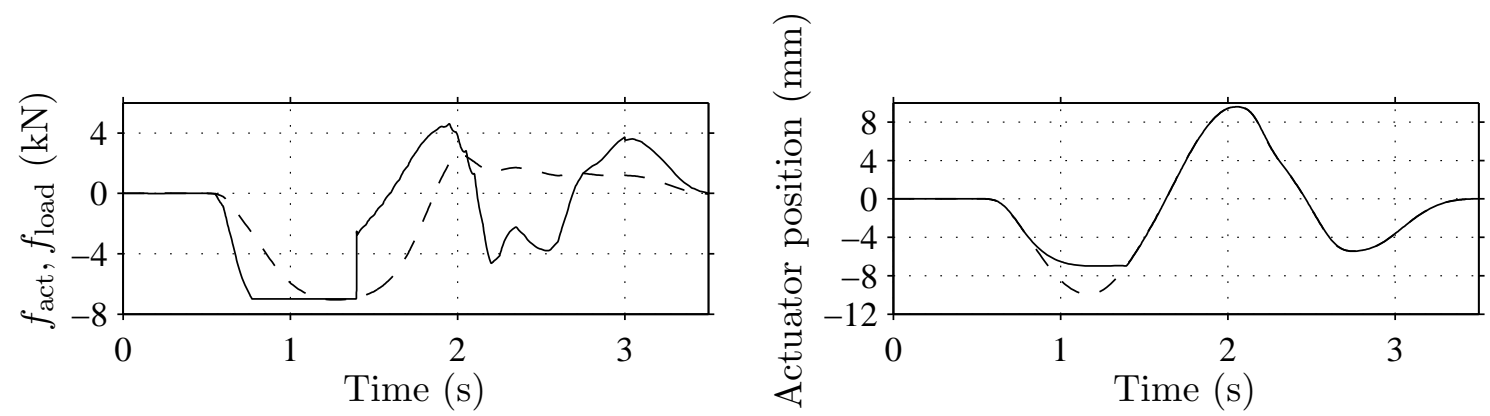

Figure 18. Steering actuator dynamics. (a) Actual steering actuator force (solid line) and load (dashed line). (b) Desired piston position (dashed line) and actual piston position (solid line).

Table 2. ACM parameters.

\begin{tabular}{lccc}
\hline Parameter & Symbol & Value & Unit \\
\hline ACM dimensions & $l_{1}, l_{2}, l_{3}, l_{4}$ & $0.22,0.205,0.290,0.020$ & $\mathrm{~m}$ \\
piston mass/damping & $\frac{m_{\text {act }}}{b_{1}}$ & $5.7471 \mathrm{e}-006$ & $\mathrm{Ns}$ \\
\hline
\end{tabular}

(a)

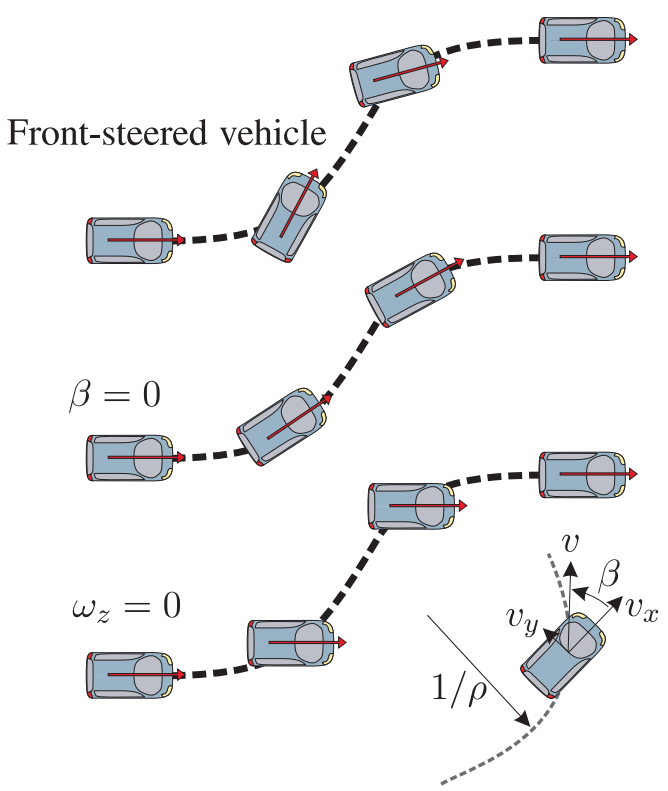

(b)
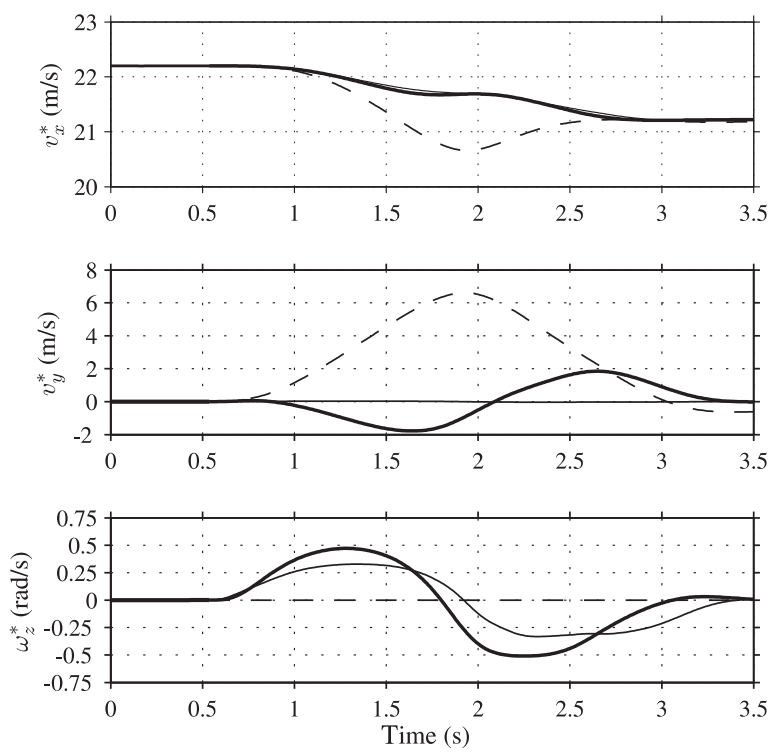

Figure 19. (a) Vehicle slip strategies maintaining the same curvature. (b) The original trajectory modified with no vehicle slip (solid line), the original trajectory modified with no vehicle yaw rate (dashed line) and the unmodified front-steered trajectory (bold line).

\section{Abandoning the conventional front-steered trajectory}

In section 4, the ACM vehicle was evaluated for the scenario where tyre forces were allocated from a conventional front-steered vehicle description. This trajectory is, to a great extent, coupled to the conventional front-steered vehicle configuration, which has many kinematics restrictions, and consequently more tyre force constraints. However, for a given driver input command, vehicle behaviour does not necessarily need to be equivalent to the conventional front-steered vehicle. One example of this, is vehicle slip, $\beta$, also referred to as the vehicle heading direction, depicted in figure 19a. For a conventional front-steered configuration $\beta$ has a significant impact on the vehicle's yaw stability $[15,16]$ and therefore is also essential for the driver's perception of balance and stability margin. However, the ACM concept has no such built-in constraint and this section presents an approach to relax the coupling between vehicle slip and yaw rate.

The original vehicle trajectory can partly be modified to follow an identical road curvature, but with different vehicle slip and vehicle yaw rate, as illustrated in figure 19b. This is accomplished by re-expressing 

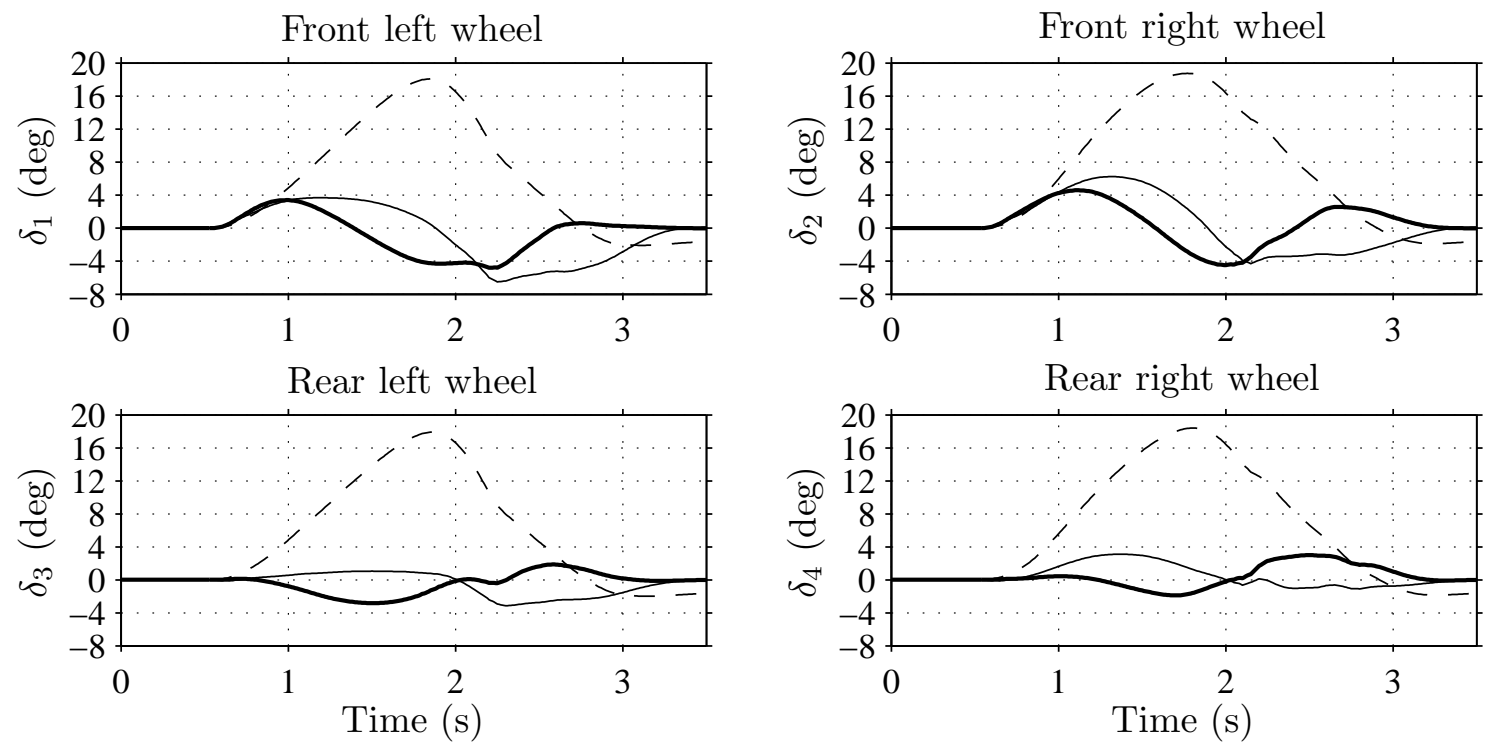

Figure 20. The resulting steering angles for the ACM vehicle with no vehicle slip present (solid line), no vehicle yaw rate present (dashed) and during conditions where the original front-steered trajectory is unchanged (bold line).

the vehicle states, $\left(v_{x}, v_{y}, \omega_{z}\right)$, to another reference frame, $(v, \beta, \rho)$, where $v$ is the vehicle velocity and $\rho$ represents the curvature in the $\mathrm{x}-\mathrm{y}$ plane, as follows

$$
v=\sqrt{v_{x}^{2}+v_{y}^{2}}, \quad \beta=\arctan \left(\frac{v_{y}}{v_{x}}\right), \quad \rho=\frac{\omega_{z}+\dot{\beta}}{v} .
$$

If $\beta^{\text {ref }}$ is chosen as the desired reference input, the new states, $\left(v_{x}^{*}, v_{y}^{*}, \omega_{z}^{*}\right)$, are found from the original states, $\left(v_{x 0}, v_{y 0}, \omega_{z 0}\right)$, as

$$
\omega_{z}^{*}=\omega_{z 0}+\frac{\mathrm{d}}{\mathrm{d} t}\left[\arctan \left(\frac{v_{y 0}}{v_{x 0}}\right)\right]-\dot{\beta}^{\mathrm{ref}}, \quad v_{x}^{*}=\left(\sqrt{v_{x 0}^{2}+v_{y 0}^{2}}\right) \cos \beta^{\mathrm{ref}}, \quad v_{y}^{*}=\left(\sqrt{v_{x 0}^{2}+v_{y 0}^{2}}\right) \sin \beta^{\mathrm{ref}} .
$$

Hence, vehicle slip can be relaxed while maintaining vehicle speed and trajectory curvature $\left(v^{*}=v_{0}\right.$ and $\left.\rho^{*}=\rho_{0}\right)$. As a result, there is an infinite set of possible trajectories through the $(v, \rho, \beta)$ space. To define a subset, which can be implemented in practice, investigations also have to involve aspects such as passenger comfort.

Here, the problem is approached by investigating trajectories that limit what is assumed to be of interest for the manoeuvre in figure $6 \mathrm{~b}$. As seen in figure 19a, the first and the latter cases generates $\beta$ with opposite signs while the middle approach keeps $\beta=0$. In figures 20, 21 and 22 , the resulting steering angles, $\delta_{i}$, in-wheel motor torques, $T_{i}$, and the tyre force potential usage, $\eta_{i}$, are shown for these different vehicle slip approaches. In these figures, $\omega_{x}$ and $\omega_{y}$ are similar and also equal to vehicle behavior presented in figure 6 and 7 , while $v_{x}, v_{y}$ and $\omega_{z}$ have been adjusted to generate the vehicle slip strategies $\beta=0$ and $\omega_{z}=0$ described in figure 19a.

The conventional trajectory implies the most yaw activity and thereby more yaw torque allocated while the latter, $\omega_{z}=0$, allows the resources available to be focused on lateral force generation. As a result, the effort spent in the latter case is less, which also is indicated in figure 22 showing a more evenly distributed use of the adhesion potential, both over the different tyres and over time. A further drawback with the original trajectory is that it forces the adhesion potential at the rear axle closer to saturation than for the front axle, which indicates a poor safety margin.

Figure 20 reveals that the zero yaw approach cannot last for long, because of the rapidly increasing steering angles, but it seems quite useful for handling initial transients and it could possibly also be used for the high-frequency driver input. From this figure it is interesting to note that the front-steered trajectory 

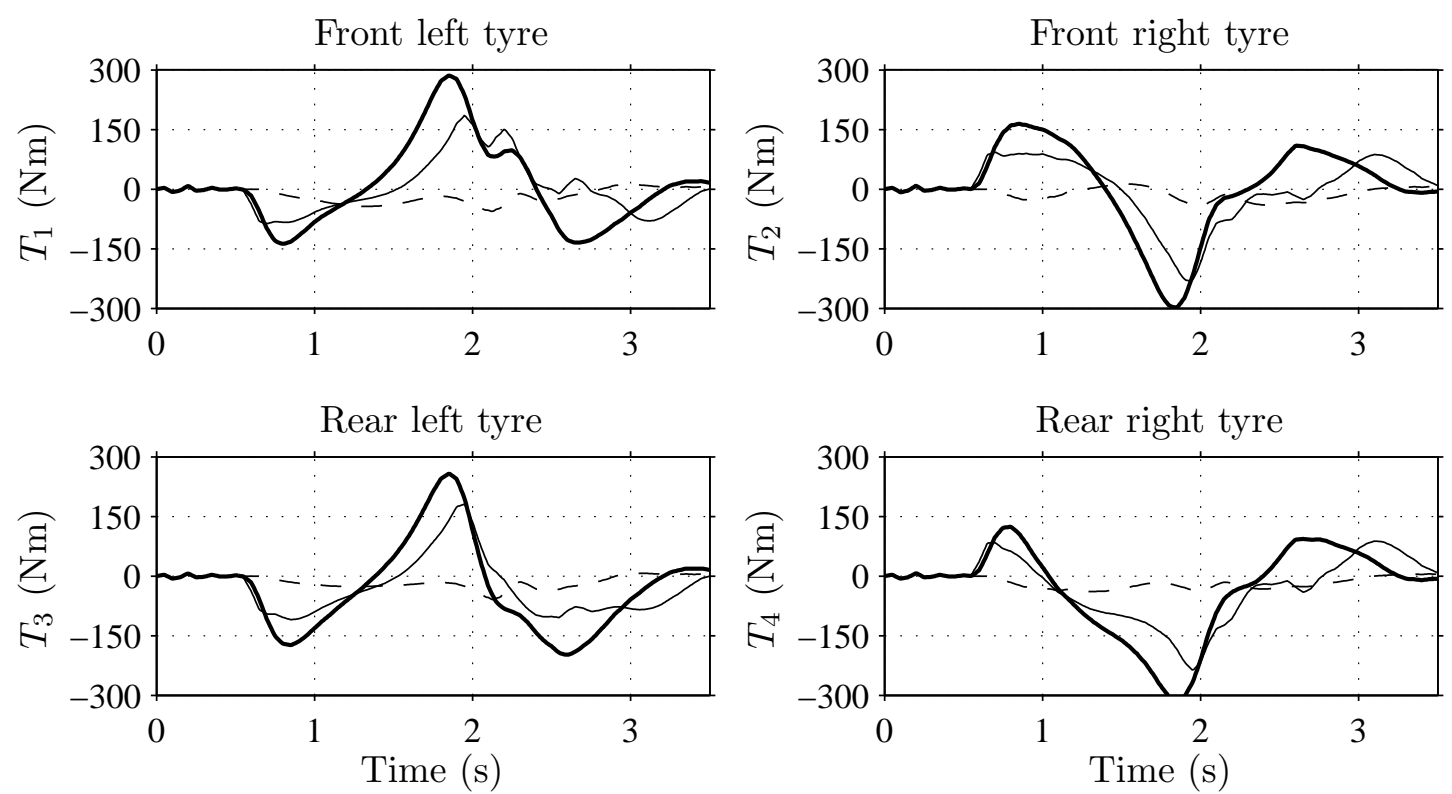

Figure 21. The resulting in-wheel motor torques for the ACM vehicle with no vehicle slip present (solid line), no vehicle yaw rate present (dashed) and during conditions where the original front-steered trajectory is unchanged (bold line and direct proportional to the graphs in figure 11).
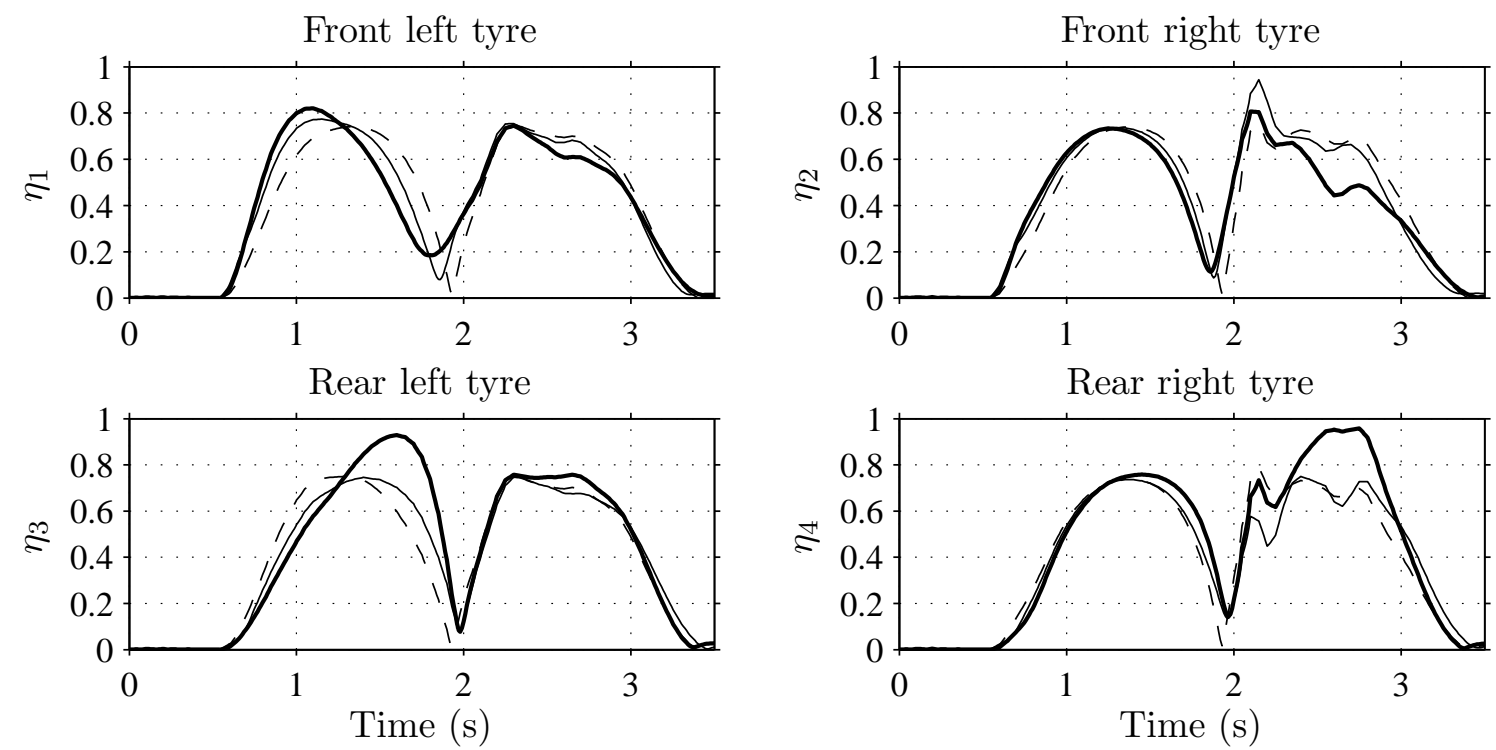

Figure 22. The resulting tyre force potential usage for the ACM vehicle with no vehicle slip present (solid line), no vehicle yaw rate present (dashed) and during conditions where the original front-steered trajectory is unchanged (bold line and equivalent to the dashed line in figure 12).

does not result in zero steering angles at the rear wheels. There are two important reasons for this; some of the required yaw moment is generated through the allocation of longitudinal forces and the resulting lateral force due to roll-induced toe and camber changes for the original vehicle must be compensated for.

$\beta=0$ represents the most common approach when relaxing $\beta$, which is seen in the majority of publications dealing with control of a vehicle with additional rear axle steering. Presumably, this is in line with driver preferences which might be explained by the driver perception being directed towards the front. This strategy lies inbetween the other strategies which also can be seen in the results. 


\section{Conclusions}

This paper has presented an approach of force allocation by optimisation with tyre force constraints and cost functions, where tyre forces are allowed to be freely controlled. This approach has been tested and evaluated using a design proposal of an autonomous corner module. A comparison with a conventional frontsteered vehicle during a transient open-loop response test has been carried out, where findings demonstrate tyre forces to be allocated differently; in-wheel motors are used to generate an additional yaw moment and the adhesion potential is better utilised, especially during low vertical load. However, in conventional vehicles, yaw rate and vehicle slip cannot be separately controlled. On the contrary, the ACM configuration is shown to be capable of performing motions, where these quantities can be individually controlled.

This paper shows that the ACM configuration can perform the same manoeuvre as the conventional front-steered vehicle studied using less tyre potential. Thus using this, there is a potentially better margin for even tougher tasks. Also, it is shown that through the additional degrees of freedom, the vehicle side slip could be relaxed which, in turn, allow for further improved performance.

From these findings, its evident that technologies, where tyre forces can be freely controlled, can be used to assign different motion patterns for a variety of driving situations. Further research should focus on how these possibilities can be utilised. This paper has also demonstrated the influence of steering actuator limitation, which is shown to restrict the desired trajectory. Finally, since the ACM, and also similar technologies, is characterised by unknown transfer functions of steering, the authors suggest to further develop and use the distribution of adhesion potential as a measure of vehicle stability.

\section{Acknowledgments}

- This work was financed in part by the Swedish National Energy Agency and the Swedish National Green Car Programme.

- The authors are grateful to Miss Catharina Hansen at Volvo Car Corporation for supplying the ADAMS/car computer simulations. In addition, we would like to thank Mr Alf Söderström at Volvo Car Corporation and Professor Annika Stensson Trigell at KTH for their immense support. Finally, the authors would like to express their gratitude to the steering committees involved for excellent guidance.

\section{References}

[1] Andreasson, J. and Laine, L., 2004, Driving dynamics for hybrid electric vehicles considering handling and control architecture, Journal of Vehicle System Dynamics, Vol. 41, pp. 497-506.

[2] Abe, M. and Mokhiamar, O., 2004, Simultaneous optimal distribution of lateral and longitudinal tire forces for the model following control, Journal of Dynamic Systems, Measurement, and Control, Vol. 126, Issue 4, pp. 753-763.

[3] Knobel, C., Pruckner, A. and Bünte, T., 2006, Optimized force allocation. A general approach to control and to investigate the motion of over-actuated vehicles, Proc. 4th IFAC Symposium on Mechatronics, Heidelberg, Germany, 12-14 September.

[4] Orend, R., 2004, Vehicle motion feedforward control with minimum utilisation of the friction potential at all four tyres, Journal of Vdi Berichte, Vol. 1828, pp. 475-484.

[5] Jonasson, M., Zetterström, S. and Trigell, A. S., 2006, Autonomous corner modules as an enabler for new vehicle chassis solutions, Proc. 31st FISITA World Automotive Conference, Yokohama, Japan, 22-27 October.

[6] Technical Committee ISO/TC 22, 1988, Road vehicles - Transient open-loop response test method with one sinusoidal input. ISO/TR 8725, 15 May.

[7] Zetterström, S., 2002, Electromechanical steering, suspension, drive and brake modules, Proc. 56th IEEE Vehicular Technology Conference, Vancouver, Canada, 24-28 September, Vol. 3, pp. 1856-1863.

[8] Fredriksson, J., Andreasson, J. and Laine, L., 2004, Wheel force distribution for improved handling in a hybrid electric vehicle using nonlinear control, Proc. 43rd IEEE Conf. on Decision and Control, Nassau, Bahamas, 14-17 December, Vol. 4, pp. 4081-4086.

[9] Valášek, M., 2002, Design and control of under-actuated and over-actuated mechanical systems - challenges of mechanics and mechatronics, Proc. 5th IACM World Congress on Computational Mechanics, Vienna, Austria, 7-12 July.

[10] He, P., 2006, Improvement of EV maneuverability and safety by disturbance observer based dynamic force distribution, Proc. 22nd International Battery, Hybrid and Fuel Cell Electric Vehicle Symposium and Exposition, Yokohama, Japan, 23-28 October.

[11] Pacejka, H. B., 2002, Tyre and vehicle dynamics, Butterworth-Heinemann, Oxford, United Kingdom.

[12] Watanahe, K. and Muramatsu, E., 2003, Error -independent stabilization of internal model control, Proc. SICE Annual Conference, Fukui University, Japan, 4-6 August.

[13] Schwarzmann, D., Nitsche, R. and Lunze, J., 2006, Diesel boost pressure control using flatness-based internal model control, Society of Automotive Engineers, No. 2006-01-0855.

[14] Doenitz, S., 1999, Comparison of disturbance suppression for servo drives, Proc. 8th European Conf. on Power Electronics and Applications, Lausanne, Switzerland, 7-9 September. 
[15] Milliken, W. F., Dell'Amico, F. and Rice, R. S., 1976, The static directional stability and control of the automobile, Society of Automotive Engineers, No. 760712.

[16] Shibahata, Y., Shimada, K. and Tomari, T., 1992, The improvement of vehicle maneuverability by direct yaw moment control, Society of Automotive Engineers of Japan, No. 928393. 


\section{Roman symbols}

a

$a_{y}$

$B_{y}$

$b$

$b_{1}$

$c_{1}$

$c_{2}$

$C_{F \alpha_{i}}$

$C_{y}$

$d_{1}, d_{2}, d_{3}, d_{4}$

$e_{\text {pitch }}$

$e_{\text {roll }}$

$f_{a_{i}}$

$f_{\text {act }}$

$f_{b_{i}}$

$f_{i}^{\prime \max }$

$f_{\text {load }}$

$f_{o_{x}}$

$f_{o_{y}}$

$f_{o_{z}}$

$f_{u_{x}}$

$f_{u_{z}}$

$f_{z, \text { nom }}$

$h$

$I_{x x}, I_{y y}, I_{z z}$

$k$

$k_{x 1}, k_{x 2}, k_{x 3}, k_{x 4}$

$k_{y 1}, k_{y 2}, k_{y 3}, k_{y 4}$

$l_{1}, l_{2}, l_{3}, l_{4}$

$m$

$m_{\text {act }}$

$m_{w}$

$n$

$r$

$T_{e_{i}}$

$T_{f_{i}}$

$T_{i}$

$t_{p}$

$v$

$v_{\text {act }}$

$v_{x}$

$v_{y}$

$v_{z}$

\section{Bold Roman symbols}

$\mathbf{A}_{\mathbf{i}}^{\prime}$

$b_{i}$

$\boldsymbol{f}=\left[f_{x_{1}} f_{y_{1}} f_{z_{1}} \cdots f_{x_{4}} f_{y_{4}} f_{z_{4}}\right]^{\mathrm{T}}$

$\boldsymbol{f}^{\prime}=\left[\begin{array}{llll}f_{x_{1}}^{\prime} f_{y_{1}}^{\prime} f_{z_{1}} \cdots f_{x_{4}}^{\prime} f_{y_{4}}^{\prime} f_{z_{4}}\end{array}\right]^{\mathrm{T}}$

$\boldsymbol{f}_{\text {glob }}=\left[\begin{array}{lllllll}F_{x} & F_{y} & F_{z} & M_{x} & M_{y} & M_{z}\end{array}\right]^{\mathrm{T}}$

$\mathbf{T}_{\mathbf{i}}$

I

$\mathbf{W}=\operatorname{diag}\left(\mathbf{w}_{\mathbf{1}}, \mathbf{w}_{\mathbf{2}}, \ldots\right)$
Tyre force limitation $(\mathrm{N})$

Lateral acceleration $\left(\mathrm{m} / \mathrm{s}^{2}\right)$

Stiffness factor

Tyre force limitation $(\mathrm{N})$

Piston damping $(\mathrm{Ns} / \mathrm{m})$

Tyre parameter

Tyre parameter

Cornering stiffness $(\mathrm{N} / \mathrm{rad})$

Shape factor

Vehicle dimensions (m)

Distance between pitch-axis and centre of gravity $(\mathrm{m})$

Distance between roll-axis and centre of gravity $(\mathrm{m})$

Steering force for inner actuator of $i$ th corner $(\mathrm{N})$

Steering actuator force $(\mathrm{N})$

Steering force for outer actuator of $i$ th corner $(\mathrm{N})$

Maximum tyre force at $i$ th tyre $(\mathrm{N})$

Steering actuator load $(\mathrm{N})$

Longitudinal force, upper arm $(\mathrm{N})$

Lateral force, upper arm $(\mathrm{N})$

Vertical force, upper arm $(\mathrm{N})$

Longitudinal force, lower arm $(\mathrm{N})$

Vertical force, lower arm (N)

Tyre parameter

Height of vehicle centre of gravity $(\mathrm{m})$

Principal moments of inertia $\left(\mathrm{kgm}^{2}\right)$

Each tangent plane in the tyre force limitation surface

Tyre parameters

Tyre parameters

ACM dimensions $(\mathrm{m})$

Vehicle mass $(\mathrm{m})$

Piston equivalent mass $(\mathrm{m})$

Unsprung mass $(\mathrm{kg})$

Number of tangent plane

Tyre radius $(\mathrm{m})$

In-wheel motor torque on $i$ th wheel $(\mathrm{Nm})$

Torque from $i$ th friction brake $(\mathrm{Nm})$

Total torque on $i$ th wheel $(\mathrm{Nm})$

Tyre pneumatic trail (m)

Vehicle velocity $(\mathrm{m} / \mathrm{s})$

Piston velocity, steering actuator $(\mathrm{m} / \mathrm{s})$

Vehicle longitudinal velocity $(\mathrm{m} / \mathrm{s})$

Vehicle lateral velocity $(\mathrm{m} / \mathrm{s})$

Vehicle vertical velocity $(\mathrm{m} / \mathrm{s})$

Tyre force limitation equipotential surface for the $i$ th tyre $(\mathrm{N})$

Vector, tyre force limitation equipotential surface for the $i$ th tyre $(\mathrm{N})$

Vehicle corner forces $(\mathrm{N})$

Tyre forces $(\mathrm{N})$

Vehicle global forces (N, Nm)

Transform matrix $i$ th wheel

Identity matrix

Prioritisation vector for use in the optimisation process 


\section{Greek symbols}

$\alpha_{i}$

$\beta$

$\beta_{f}$

$\beta_{r}$

$\gamma$

$\delta_{i}$

$\delta_{\text {swa }}$

$\eta_{i}$

$\kappa_{i}$

$\mu_{i}$

$\rho$

$\varphi$

$\omega_{x}$

$\omega_{y}$

$\omega_{z}$

\section{Subscript and superscript}

$*$

cost

glob

ref

0

1,2

3,4
Side slip angle of $i$ th tyre (rad)

Vehicle slip (rad)

Front axle slip (rad)

Rear axle slip (rad)

Wheel camber (rad)

Steering angle of $i$ th tyre (rad)

Steering wheel angle (rad)

Adhesion potential on the $i$ th tyre

Longitudinal slip

Friction coefficient of $i$ th tyre

Path curvature $(1 / \mathrm{m})$

Vehicle roll angle ( $\mathrm{rad})$

Vehicle roll rate $(\mathrm{rad} / \mathrm{s})$

Vehicle pitch rate $(\mathrm{rad} / \mathrm{s})$

Vehicle yaw rate $(\mathrm{rad} / \mathrm{s})$

Modified vehicle states

Cost functions in the optimisation process $(\mathrm{N})$

Global forces acting on centre of gravity (N)

Reference signals

Original vehicle states

Front left and right tyre

Rear left and right tyre

Denote tyre forces in the wheel coordinates 